\title{
Optical Parametric Generator based on Orientation-patterned Gallium Phosphide
}

\author{
Hanyu Ye ${ }^{1}$, S. Chaitanya Kumar ${ }^{1,2}$, Junxiong Wei ${ }^{1}$, P. G. Schunemann ${ }^{3}$, M. Ebrahim-Zadeh ${ }^{1,2,4}$ \\ 1. ICFO-Institut de Ciencies Fotoniques, The Barcelona Institute of Science and Technology, 08860 Castelldefels (Barcelona), Spain \\ 2. Radiantis, Polígon Camí Ral, 08850 Gavà, Barcelona, Spain \\ 3. BAE Systems, Incorporated, MER15-1813, P.O. Box 868, Nashua, New Hampshire 03061-0868, USA \\ 4. ICREA, Passeig Lluis Companys 23, 08010 Barcelona, Spain \\ Corresponding author: chaitanya.suddapalli@icfo.es
}

\begin{abstract}
:
We report the first pulsed optical parametric generator based on Orientation-patterned Gallium Phosphide. The output is tunable from 1721-1850 nm (signal) and 2504-2787 nm (idler), providing a total output power of $18 \mathrm{~mW}$.
\end{abstract}

Non-oxide nonlinear crystals are of great significance in developing optical parametric coherent sources in the mid-infrared (mid-IR) region beyond $4 \mu \mathrm{m}$. The newly developed nonlinear semiconductor material, orientationpatterned Gallium Phosphide (OP-GaP), is promising to be the next-generation nonlinear medium because of its high nonlinearity $\left(d_{14}=70.6 \mathrm{pm} / \mathrm{V}\right)$, quasi-phase-matching $(\mathrm{QPM})$ structure and significantly lower absorption in the near-IR region [1] as compared to other mid-IR materials, which make it possible to deploy well established $1-\mu \mathrm{m}$ lasers for pumping optical parametric devices to generate deep mid-IR radiation. Here, we built and characterized the first optical parametric generator (OPG) based on OP-GaP, pumped by a pulsed Nd:YAG laser.

The schematic of the experimental setup for the OPG is shown in Fig. 1(a). A Q-switched Nd:YAG laser operating at $25 \mathrm{kHz}$ repetition rate is used as the pump source. The nonlinear crystal for OPG is a 40-mm-long OP-GaP crystal with a single grating period of $15.5 \mu \mathrm{m}$, mounted in an oven to accomplish wavelength tuning by changing the temperature. The pump beam was focused into the OP-GaP crystal with a beam waist radius of $\sim 89$ $\mu \mathrm{m}$ for parametric gain. The vertical polarization was chosen by rotating a half-wave plate to maximize the down-conversion efficiency. We performed both experimental measurement and theoretical calculation for investigating the temperature tuning characteristics of OP-GaP. The measured data covering the wavelength range of $1721 \mathrm{~nm}-1850 \mathrm{~nm}$ (signal) and $2504 \mathrm{~nm}-2787 \mathrm{~nm}$ (idler) for an operating temperature varying from $50^{\circ}-180^{\circ} \mathrm{C}$ shows a good agreement with the calculation using the relevant Sellmeier equations [2] as presented in Fig. 1(b). The total power and idler power directly measured using two long pass filters (Edmund Optics, 68652,68653 ) across the range of $50-160^{\circ} \mathrm{C}$, as well as the estimated signal power, are shown in Fig. 1(c), for a fixed pump power of $2 \mathrm{~W}$. The highest powers reached $18.1 \mathrm{~mW}$ (total, $110^{\circ} \mathrm{C}$ ), $13.1 \mathrm{~mW}$ (signal, $110^{\circ} \mathrm{C}$ ) and $5.06 \mathrm{~mW}$ (idler, $120^{\circ} \mathrm{C}$ ). The transmission of the pump across the temperature range is also recorded as shown in Fig.1 (c), which dramatically decreased from $26.7 \%$ at $50{ }^{\circ} \mathrm{C}$ to $18.4 \%$ at $160{ }^{\circ} \mathrm{C}$ revealing a stronger absorption of the crystal at the higher temperature. This increasing absorption could be responsible for the power drop towards higher temperatures. Furthermore, we performed the power scaling measurement for the signal and idler at $120^{\circ} \mathrm{C}$, which is shown in Fig. 1(d). The signal and idler power reached $12.6 \mathrm{~mW}$ at $1769 \mathrm{~nm}$ and 5.2 $\mathrm{mW}$ at $2670 \mathrm{~nm}$ with slope efficiencies of $1.23 \%$ and $0.51 \%$ respectively. The relevant transmission of the used pump is also given in Fig. 1(d), which shows a slight decrease in the region between $25.4 \%$ and $23.6 \%$ when increasing the pump power. In a separate experiment, we have performed detailed studies of material damage threshold in OP-GaP using the same pulsed Nd:YAG laser and estimated it to be $\sim 0.8 \mathrm{~J} / \mathrm{cm}^{2}$.
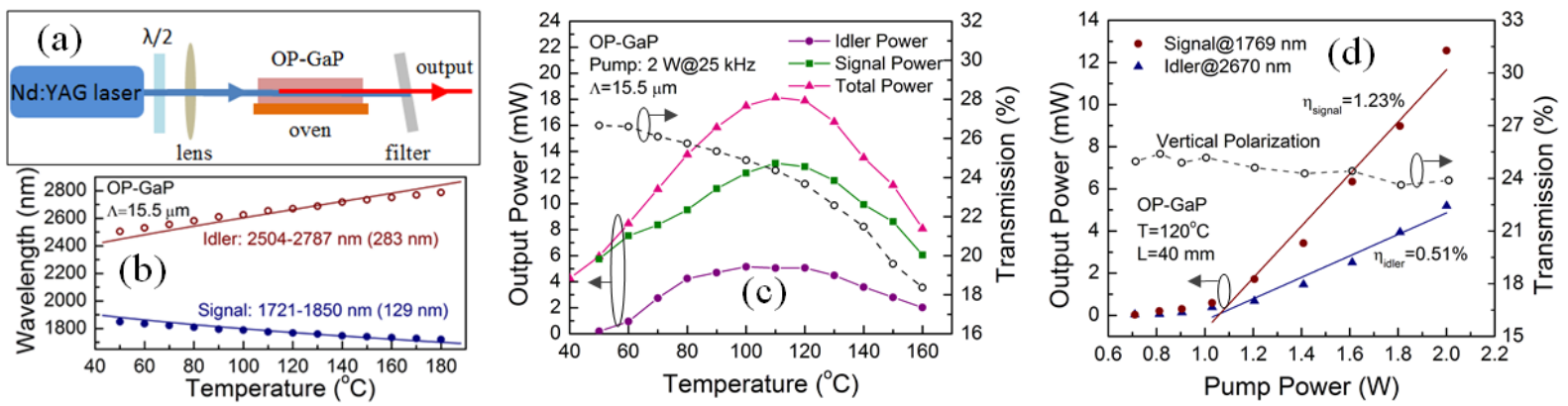

Fig. 1 (a) Experimental setup of the OPG, (b) Calculated temperature tuning curves and experimental data, (c) Output power and pump transmission across tuning range, (d) Power scaling of the signal and idler.

In summary, we have demonstrated for the first time we believe a pulsed OPG based on an OP-GaP crystal. The OPG output is temperature tunable from 1721 to $1850 \mathrm{~nm}$ for the signal along with 2504$2787 \mathrm{~nm}$ for the idler, which could be further extended into deeper mid-IR region by using OP-GaP 
crystals with longer grating periods. We also experimentally studied the performance of the OPG output and the used OP-GaP crystal, paving the way for the development of 1- $\mu \mathrm{m}$ laser pumped deep mid-IR parametric sources. Complete characterization of the OPG including stability and mid-IR beam quality will be presented.

\section{References:}

[1] P. G. Schunemann, K. T. Zawilski, L. A. Pomeranz, D. J. Creeden, and P. A. Budni, J. Opt. Soc. Am. B: Opt. Phys. 33, D36-D43 (2016). [2] P. G. Schunemann, L. A. Pomeranz, D. J. Magarrell, J. C. MeCarthy, K. T. Zawilski, D. E. Zelmon, CLEO: 2015 (OSA), SW3O.4 (2015). 